\title{
Stacked GaAsSbN-capped InAs/GaAs quantum dots for enhanced solar cell efficiency
}

\author{
A.D. Utrilla, J.M. Ulloa, Ž. Gačević, A. Guzman and A. Hierro \\ ISOM and Dpto.Ing.Electrónica, Universidad Politécnica de Madrid, Spain
}

Different approaches have recently arisen aiming to exceed the ShockleyQueisser efficiency limit. Particularly, the use of self-organized quantum dots (QD) has been recently proposed in order to introduce new states within the barrier material, which enhances the sub-band gap absorption yielding a photocurrent increase. Stacking QD layers allows exploiting their unique properties for intermediate-band solar cells (SC) or tandem cells. In all these cases, tuning the QD properties by modifying the capping layer $(\mathrm{CL})$ can be very useful.

In this respect, the beneficial impact of thin GaAsSb CLs on InAs/GaAs QD properties is already well known. ${ }^{1}$ Indeed, using stacks of GaAsSb-capped QDs has recently been found to improve intermediate band SC characteristics. ${ }^{2}$ However, a limitation is found regarding the number of layers that can be stacked due to the strain accumulation. On the other hand, introducing $\mathrm{N}$ compensates the accumulated strain allowing the stacking of a larger number of QD layers. ${ }^{3}$ Therefore, using GaAsSbN as a CL could take advantage of both approaches. Moreover, this system allows an independent control of the QD-CL conduction and valence band offsets, ${ }^{4}$ which would allow designs that facilitate carrier extraction from the QDs.

In this work, we have studied the impact of using GaAsSbN CLs on the absorption characteristics of InAs/GaAs QDs. All the samples were grown by solid source molecular beam epitaxy on $n+G a A s$ substrates. Stacked-QD layers were embedded in the intrinsic region of a p-i-n junction for device processing.

The PL emission of the GaAsSbN-containing samples with 10 QD layers is comparable to that of the ternary counterparts (Fig. 1(a)), and the FWHM seems to correlate with the accumulated strain. X-ray diffraction (XRD) $\Theta / 2 \Theta$ scans reveal a high periodicity and a good crystal quality of the grown superlattice (SL). Reciprocal space mapping (RSM) reveals that the SL grows pseudomorphically on the underneath GaAs buffer and confirms a reduction of the accumulated strain, when compared to the GaAsSb case. Photocurrent measurements for GaAsSband GaAsSbN-containing samples are shown in figure 2. The absorption from the QDs, as well as from higher energy states possibly corresponding to the capping and wetting layers can be observed, besides that of the bulk GaAs material. A more efficient photocurrent in the long wavelength region was found when using GaAsSbN.

Since GaAsSbN growth conditions were found to be critical, different growth approaches will be analyzed. In addition, the impact of modifying the GaAs spacer thickness, the number of stacked layers, and the QD-CL band alignment will be also discuss, as well as the effect of rapid thermal annealing on the structures.

Presenting student - Corresponding author : Antonio D. Utrilla - utrilla@isom.upm.es 


\section{References}

${ }^{1}$ J. M. Ulloa, R. Gargallo-Caballero, M. Bozkurt, M. del Moral, A. Guzmán, P. M. Koenraad, and A. Hierro, Phys. Rev. B 81, 165305 (2010)

${ }^{2}$ W-S. Liu, H-M. Wu, F-H. Tsa, T-L. Hsu, and J-I. Chyi, Sol. Energy Mater. Sol. Cells 105, 237(2012)

${ }^{3}$ Ryuji Oshima, Ayami Takata, and Yoshitaka Okada, Appl. Phys. Lett. 93, 083111 (2008)

${ }^{4}$ J. M. Ulloa, D. F. Reyes, M. Montes, K. Yamamoto, D. L. Sales, D. González, A. Guzman, and A. Hierro, Appl. Phys. Lett. 100, 013107 (2012)

\section{Figures}
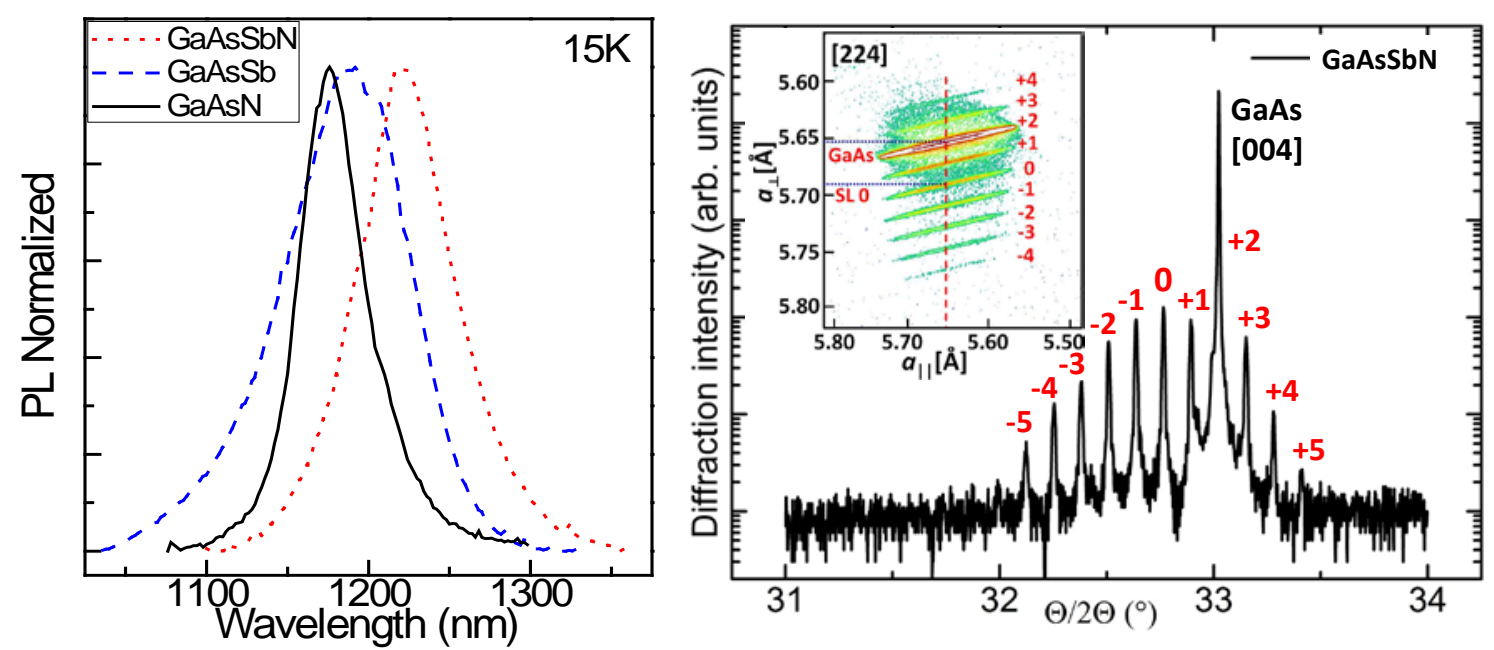

Figure 1: (a) Low temperature photoluminescence from GaAsSb-, GaAsN- and GaAsSbNcontaining samples. (b) $A \theta / 2 \theta X R D$ scan, around 004 Bragg reflection performed on the GaAsSbN-containing sample, reveals satellite peaks resolved up to the 5 th order, indicating a high periodicity and a good crystal quality of the grown SL. (Inset) RSM around 224 Bragg reflection, reveals pseudomorphic growth and a reduction of strain accumulated in the SL.

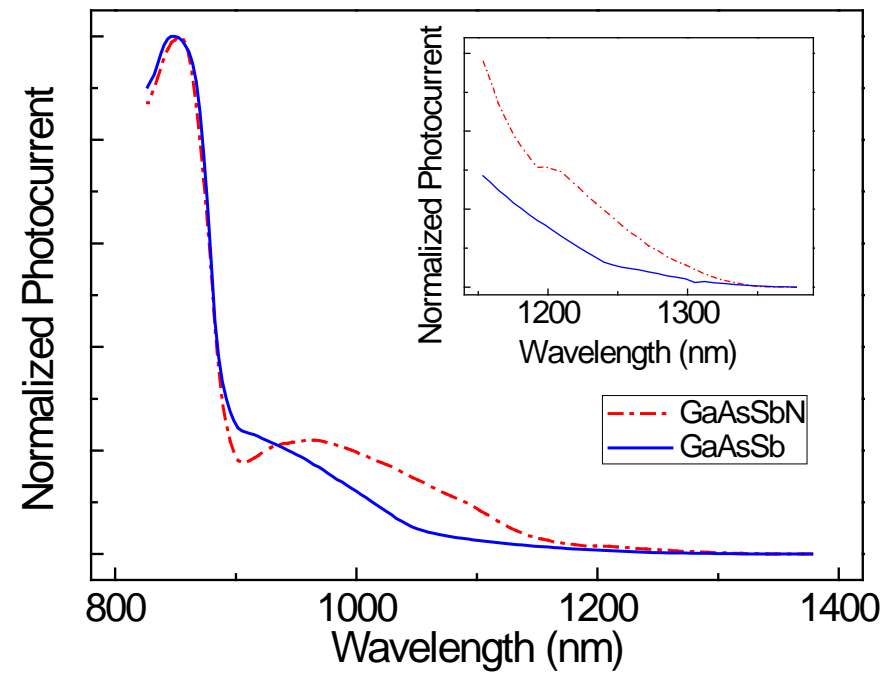

Figure 2: Room temperature photocurrent spectra for GaAsSb- and GaAsSbN-containing samples. The inset shows a zoom of the spectra around the $Q D$ absorption wavelengths. 